\title{
Acoustical Modeling of Laminated Composite Cylindrical Double-Walled Shell Lined with Porous Materials
}

\author{
K. Daneshjou, H. Ramezani and R. Talebitooti \\ Additional information is available at the end of the chapter
}

http://dx.doi.org/10.5772/48646

\section{Introduction}

Although the researchers have done many efforts to perform the numerical model such as FEM (Finite Elements Method) to investigate the wave prorogation through the shells, the analytical vibro-acoustic modeling of the composite shells is unavoidable because of the accuracy of the model in a broadband frequency. Bolton et. al. [1] investigated sound transmission through sandwich structures lined with porous materials and following Lee et. al. [2] proposed a simplified method to analyze curved sandwich structures. Daneshjou et. al. [3-5] studied an exact solution to estimate the transmission loss of orthotropic and laminated composite cylindrical shells with considering all three displacements of the shell. Recently the authors [6] have presented an exact solution of free harmonic wave propagation in a double-walled laminated composite cylindrical shell whose walls sandwich a layer of porous material using an approximate method. This investigation is focused on sound transmission through the sandwich structure, which includes the porous material core between the two laminated composite cylindrical shells to predict the reliable results for all structures used foam as an acoustic treatment.

Wave propagation through a composite cylindrical shell lined with porous materials is investigated, based on classical laminated theory. The porous material is completely modeled using elastic frame. The vibro-acoustic equations of the shell are derived considering both the shell vibration equations and boundary conditions on interfaces. These coupled equations are solved simultaneously to calculate the Transmission Loss (TL). Moreover, the results are verified with a special case where the porosity approaches zero. Finally, the numerical results are illustrated to properly study the geometrical and physical properties of composite and porous material. In addition, the effects of the stacking sequence of composite shells and fiber directions are properly studied. 


\section{Propagation of sound in porous media}

If the porous material is assumed a homogeneous aggregate of the elastic frame and the fluid trapped in pores, its acoustic behavior can be considered by the following two wave equations (See Eq. (22) and Eq. (25) of [1]):

$$
\begin{gathered}
\nabla^{4} e_{S}+A_{1} \nabla^{2} e_{S}+A_{2} e_{s}=0 \\
\nabla^{2} \varpi+\xi_{t}^{2} \varpi=0
\end{gathered}
$$

Eqs. (1) and (2) determine 2 elastic longitudinal waves and 1 rotational wave, respectively. In Eqs. (1) and (2) $\nabla$ is the vector differential operator, $e_{s}=\nabla \cdot \overline{\mathbf{u}}$ is the solid volumetric strain, $\overline{\mathbf{u}}$ is the displacement vector of the solid, $\varpi=\nabla \times \overline{\mathbf{u}}$ is the rotational strain in the solid phase, $A_{1}=\omega^{2}\left(\varphi \hat{\rho}_{22}+\mu \hat{\rho}_{11}-2 \chi \hat{\rho}_{12}\right) /\left(\varphi \mu-\chi^{2}\right), A_{2}=\omega^{4}\left(\hat{\rho}_{22} \hat{\rho}_{11}-\left(\hat{\rho}_{12}\right)^{2}\right) /\left(\varphi \mu-\chi^{2}\right)$, and $\xi_{t}$ is the wave number of the shear wave (See Eq. 10). $\hat{\rho}_{11}, \hat{\rho}_{12}$ and $\hat{\rho}_{22}$ are equivalent masses given by:

$$
\begin{gathered}
\hat{\rho}_{11}=\rho_{1}+\rho_{a}-j \sigma_{r} \phi^{2}\left(\frac{1}{\omega}+\frac{4 j \alpha_{\infty}^{2} \kappa_{v} \rho_{0}}{\sigma_{r}^{2} \Lambda^{2} \phi^{2}}\right) \\
\hat{\rho}_{12}=-\rho_{a}+j \sigma_{r} \phi^{2}\left(\frac{1}{\omega}+\frac{4 j \alpha_{\infty}^{2} \kappa_{v} \rho_{0}}{\sigma_{r}^{2} \Lambda^{2} \phi^{2}}\right) \\
\hat{\rho}_{22}=\phi \rho_{0}+\rho_{a}-j \sigma_{r} \phi^{2}\left(\frac{1}{\omega}+\frac{4 j \alpha_{\infty}^{2} \kappa_{v} \rho_{0}}{\sigma_{r}^{2} \Lambda^{2} \phi^{2}}\right)
\end{gathered}
$$

where $j$ is the imaginary unit $j^{2}=-1 . \rho_{1}$ and $\rho_{0}$ are the densities of the solid and fluid parts of the porous material. Moreover, parameters $\alpha_{\infty}, \kappa_{v}, \Lambda, \sigma_{r}, \phi$ and $\omega$ are tortuosity, air viscosity, viscous characteristic length, flow resistivity, porosity, and angular frequency, respectively. $\chi, \varphi$ and $\mu$ represent material properties: $\chi=(1-\phi) G, \mu=\phi G, \varphi=A+2 \delta$, $\delta=E / 2(1+v)$ and $A=v E /(1+v)(1-2 v) . E$ and $v$ are the in vacuo Young's modulus and Poisson's ratio of the bulk solid phase, respectively. Assuming that pores are shaped in cylindrical form, an expression for $G$ is:

$$
G=\rho_{0} c_{2}^{2}\left\{1+\left[(\varsigma-1) \sqrt{\phi \sigma_{r}} / N_{\operatorname{Pr}}^{0.5} \sqrt{-2 j \omega \rho_{0} \alpha_{\infty}}\right]\left[\frac{J_{1}\left(2 N_{\operatorname{Pr}}^{0.5} \sqrt{-2 \omega \rho_{0} \alpha_{\infty} j / \phi \sigma_{r}}\right)}{J_{0}\left(2 N_{\operatorname{Pr}}^{0.5} \sqrt{-2 \omega \rho_{0} \alpha_{\infty} j / \phi \sigma_{r}}\right)}\right]\right\}^{-1}
$$

where $\varsigma$ is the ratio of specific heats, $c_{2}$ is the speed of sound in the fluid phase of porous materials, $N_{\operatorname{Pr}}$ represents the Prandtl number, and $J_{0}$ and $J_{1}$ are Bessel functions of the first kind, zero and first order, respectively. $\rho_{a}$ is the inertial coupling term: 


$$
\rho_{a}=\phi \rho_{0}\left(\alpha_{\infty}-1\right)
$$

The complex wave numbers of the two compression (longitudinal) waves, $\xi_{\alpha}$ and $\xi_{\beta}$ are:

$$
\begin{aligned}
& \xi_{\alpha}^{2}=\frac{\omega^{2}}{2\left(\varphi \mu-\chi^{2}\right)}\left(\varphi \hat{\rho}_{22}+\mu \hat{\rho}_{11}-2 \chi \hat{\rho}_{12}+\sqrt{\wp}\right) \\
& \xi_{\beta}^{2}=\frac{\omega^{2}}{2\left(\varphi \mu-\chi^{2}\right)}\left(\varphi \hat{\rho}_{22}+\mu \hat{\rho}_{11}-2 \chi \hat{\rho}_{12}-\sqrt{\wp}\right)
\end{aligned}
$$

where

$$
\wp=\left(\varphi \hat{\rho}_{22}+\mu \hat{\rho}_{11}-2 \chi \hat{\rho}_{12}\right)^{2}-4\left(\varphi \mu-\chi^{2}\right)\left(\hat{\rho}_{22} \hat{\rho}_{11}-\hat{\rho}_{12}^{2}\right)
$$

and the wave number of the shear (rotational) wave is:

$$
\xi_{t}^{2}=\frac{\omega^{2}}{\delta}\left(\frac{\hat{\rho}_{22} \hat{\rho}_{11}-\hat{\rho}_{12}^{2}}{\hat{\rho}_{22}}\right)
$$

$\delta$ is the shear modulus of the porous material.

\section{Simplified method}

As the full method is too complicated to model the porous layer in the curved sandwich structures, thus a simplified method is expanded for this category of structures [2]. The foundation of this approximate method considers the strongest wave between those ones. It includes two steps. At the first step a flat double laminated composite with infinite extents with the same cross sectional construction is considered using the full method. Then, only the strongest wave number is chosen from the results and the material is modeled using the wave number and its corresponding equivalent density. Thus, the material is modeled as an equivalent fluid.

The strain energy which is related to the displacement in the solid and fluid phases can be defined for each wave component. The energy terms can be represented as follows; $E_{1 s}$ and $E_{1 f}$ for the airborne wave, $E_{3 s}$ and $E_{3 f}$ for the frame wave and $E_{5 s}$ for the shear wave, which the subscripts $s$ and $f$ represent the solid and fluid phase, respectively. For each new problem, comparing the ratios of the energy carried by the frame wave and the shear wave to the airborne wave in the fluid and solid phases: i.e., $\frac{E_{1 f}}{E_{1 f}}, \frac{E_{1 s}}{E_{1 f}}, \frac{E_{3 f}}{E_{1 f}}, \frac{E_{3 s}}{E_{1 f}}, \frac{E_{5 s}}{E_{1 f}}$ show the strongest wave component in the entire frequency range. 


\section{Model specification}

Figure 1 shows a schematic of the cylindrical double shell of infinite length subjected to a plane wave with an incidence angle $\gamma$. The radii and the thicknesses of the shells are $R_{i, e}$ and $h_{i, e}$ in which the subscripts $i$ and $e$ represent the inner and outer shells. A concentric layer of porous material is installed between the shells. The acoustic media in the outside and the inside of the shell are represented by density and speed of sound: $\left(s_{1}, c_{1}\right)$ outside and $\left(s_{3}, c_{3}\right)$ inside.
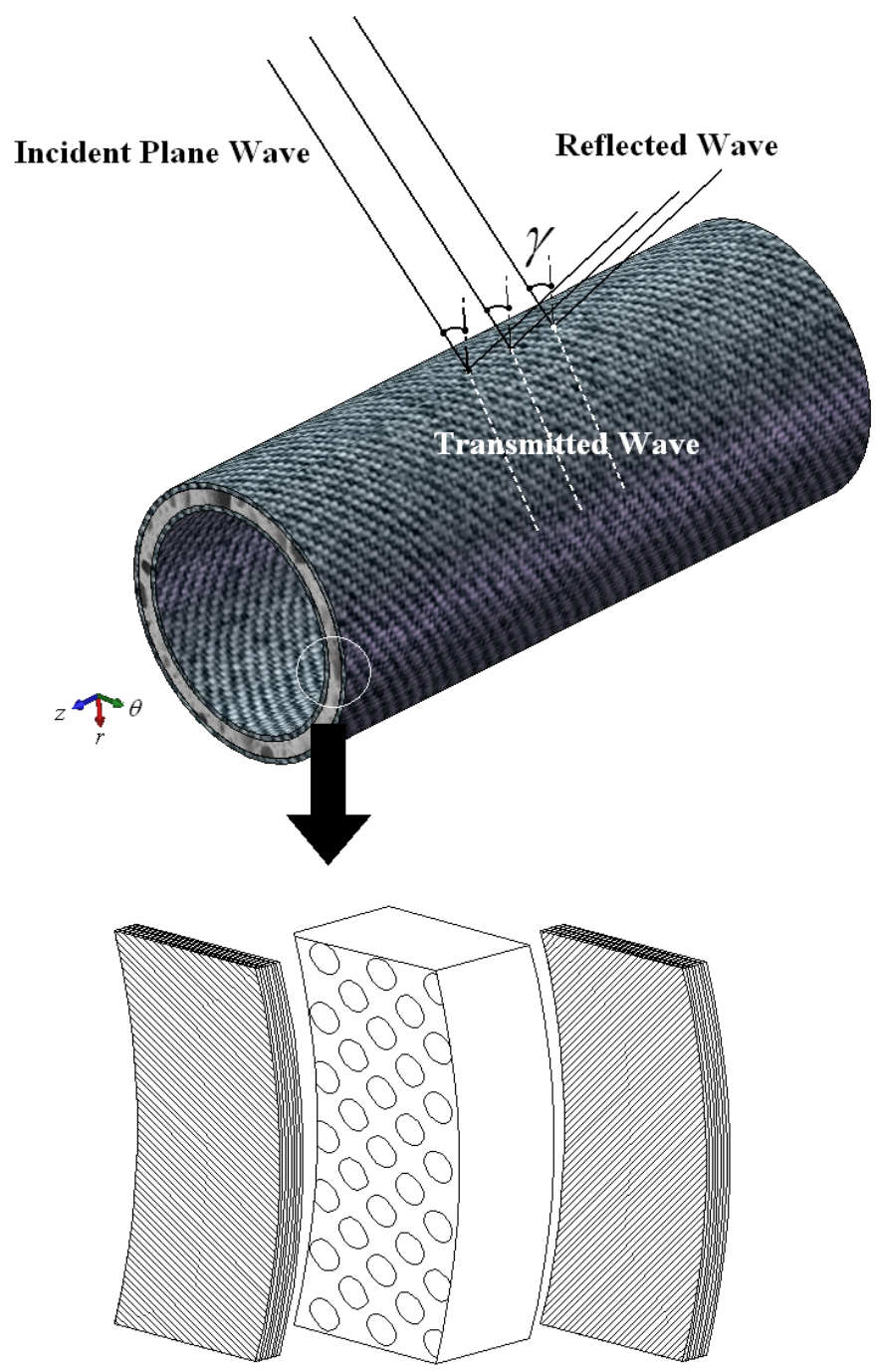

Inner Shell Porous Core Outer Shell

Figure 1. Schematic diagram of the double-walled cylindrical composite shell lined with porous materials 


\section{Applying full method to two-dimensional problem}

For a two-dimensional problem as shown in the $x-y$ plane of Fig. 2, the potential of the incident wave can be expressed as [1]:

$$
\mathfrak{I}_{i}=e^{-j\left(\xi_{x} x+\xi_{1 y} y\right)}
$$

where $\xi_{x}=\xi_{1} \sin \gamma, \xi_{1 y}=\xi_{1} \cos \gamma, \xi_{1}=\omega / c_{1}, c_{1}$ is the speed of sound in incident Medium, $\gamma$ is the angle of incidence.

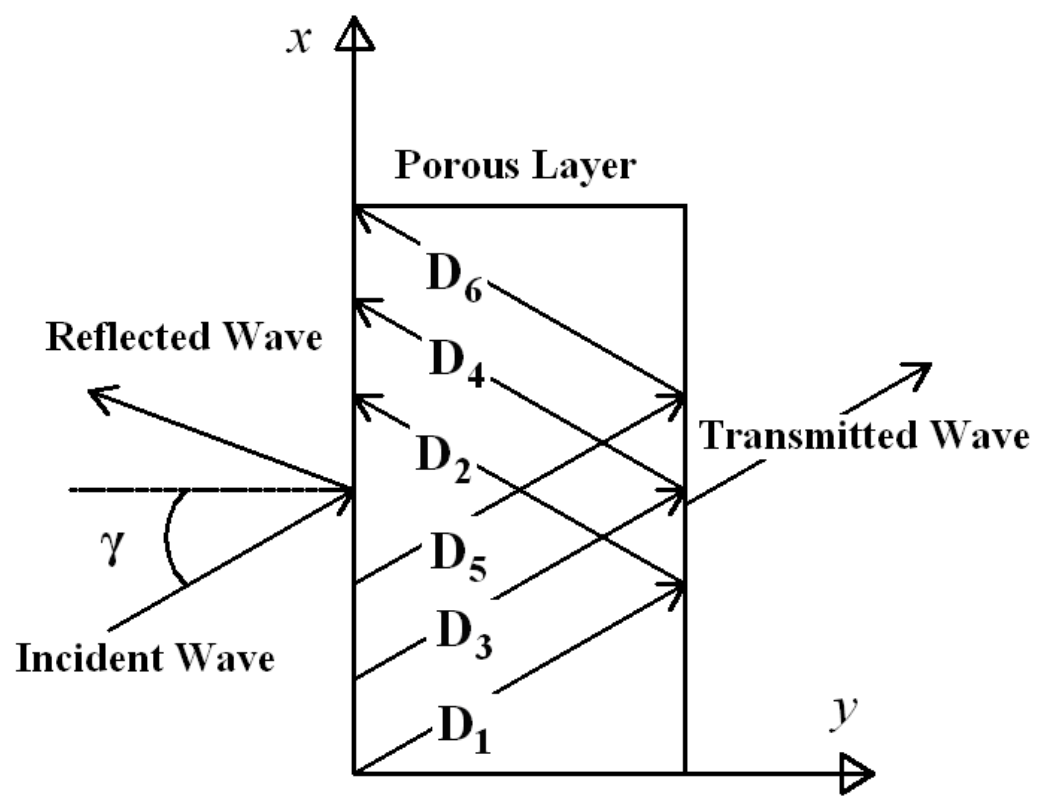

Figure 2. Illustration of wave propagation in the porous layer

Three kinds of the waves propagate in porous material, therefore six traveling waves, which have the same trace wave numbers, are induced by an oblique incident wave in a finite depth layer of porous material, as shown in Fig. 2. The $x$ - and $y$ - direction components of the displacements and stresses of the solid and fluid phases were derived by Bolton et. al. [1]. The displacements in the solid phase are:

$$
\begin{aligned}
\hat{u}_{x}=j \xi_{x} e^{-j \xi_{x} x} & {\left[\frac{D_{1}}{\xi_{\alpha}^{2}} e^{-j \xi_{\alpha y} y}+\frac{D_{2}}{\xi_{\alpha}^{2}} e^{j \xi_{\alpha y} y}+\frac{D_{3}}{\xi_{\beta}^{2}} e^{-j \xi_{\beta y} y}+\frac{D_{4}}{\xi_{\beta}^{2}} e^{j \xi_{\beta y} y}\right] } \\
& -j \frac{\xi_{t y}}{\xi_{t}^{2}} e^{-j \xi_{x} x}\left[D_{5} e^{-j \xi_{t y} y}-D_{6} e^{j \xi_{t y} y}\right]
\end{aligned}
$$




$$
\begin{gathered}
\hat{u}_{y}=j e^{-j \xi_{x} x}\left[\frac{\xi_{\alpha y}}{\xi_{\alpha}^{2}} D_{1} e^{-j \xi_{\alpha y} y}-\frac{\xi_{\alpha y}}{\xi_{\alpha}^{2}} D_{2} e^{j \xi_{\alpha y} y}+\frac{\xi_{\beta y}}{\xi_{\beta}^{2}} D_{3} e^{-j \xi_{\beta y} y}-\frac{\xi_{\beta y}}{\xi_{\beta}^{2}} D_{4} e^{j \xi_{\beta y} y}\right] \\
+j \frac{\xi_{x}}{\xi_{t}^{2}} e^{-j \xi_{x} x}\left[D_{5} e^{-j \xi_{t y} y}+D_{6} e^{j \xi_{t y} y}\right]
\end{gathered}
$$

The displacements in the fluid phase are:

$$
\begin{gathered}
\hat{U}_{x}=j \xi_{x} e^{-j \xi_{x} x}\left[b_{1} \frac{D_{1}}{\xi_{\alpha}^{2}} e^{-j \xi_{\alpha y} y}+b_{1} \frac{D_{2}}{\xi_{\alpha}^{2}} e^{j \xi_{\alpha y} y}+b_{2} \frac{D_{3}}{\xi_{\beta}^{2}} e^{-j \xi_{\beta y} y}+b_{2} \frac{D_{4}}{\xi_{\beta}^{2}} e^{j \xi_{\beta y} y}\right] \\
-j g \frac{\xi_{t y}}{\xi_{t}^{2}} e^{-j \xi_{x} x}\left[D_{5} e^{-j \xi_{t y} y}-D_{6} e^{j \xi_{t y} y}\right] \\
\hat{U}_{y}=j e^{-j \xi_{x} x}\left[b_{1} \frac{\xi_{\alpha y}}{\xi_{\alpha}^{2}} D_{1} e^{-j \xi_{\alpha y} y}-b_{1} \frac{\xi_{\alpha y}}{\xi_{\alpha}^{2}} D_{2} e^{j \xi_{\alpha y} y}+b_{1} \frac{\xi_{\beta y}}{\xi_{\beta}^{2}} D_{3} e^{-j \xi_{\beta y} y}-b_{1} \frac{\xi_{\beta y}}{\xi_{\beta}^{2}} D_{4} e^{j \xi_{\beta y} y}\right] \\
+j g \frac{\xi_{x}}{\xi_{t}^{2}} e^{-j \xi_{x} x}\left[D_{5} e^{-j \xi_{t y} y}+D_{6} e^{j \xi_{t y} y}\right]
\end{gathered}
$$

The stresses in the solid phase are:

$$
\begin{aligned}
& \hat{\sigma}_{y}^{s}=e^{-j \xi_{x} x}\left[\begin{array}{l}
\left(2 \delta \frac{\xi_{\alpha y}^{2}}{\xi_{\alpha}^{2}}+A+b_{1} \chi\right) D_{1} e^{-j \xi_{\alpha y} y}+\left(2 \delta \frac{\xi_{\alpha y}^{2}}{\xi_{\alpha}^{2}}+A+b_{1} \chi\right) D_{2} e^{j \xi_{\alpha y} y} \\
\left.+\left(2 \delta \frac{\xi_{\beta y}^{2}}{\xi_{\beta}^{2}}+A+b_{2} \chi\right) D_{3} e^{-j \xi_{\beta y} y}+\left(2 \delta \frac{\xi_{\beta y}^{2}}{\xi_{\beta}^{2}}+A+b_{2} \chi\right) D_{4} e^{j \xi_{\beta y} y}\right] \\
+2 \delta \frac{\xi_{x} \xi_{t y}}{\xi_{t}^{2}}\left(D_{5} e^{-j \xi_{t y} y}-D_{6} e^{j \xi_{t y} y}\right)
\end{array}\right] \\
& \hat{\tau}_{x y}=e^{-j \xi_{x} x} \delta\left[\begin{array}{l}
\frac{2 \xi_{x} \xi_{\alpha y}}{\xi_{\alpha}^{2}}\left(D_{1} e^{-j \xi_{\alpha y} y}-D_{2} e^{j \xi_{\alpha y} y}\right)+\frac{2 \xi_{x} \xi_{\beta y}}{\xi_{\beta}^{2}}\left(D_{3} e^{-j \xi_{\beta y} y}-D_{4} e^{j \xi_{\beta y} y}\right) \\
+\frac{\left(\xi_{x}^{2}-\xi_{t y}^{2}\right)}{\xi_{t}^{2}}\left(D_{5} e^{-j \xi_{t y} y}+D_{6} e^{j \xi_{t y} y}\right)
\end{array}\right]
\end{aligned}
$$

The stresses in the fluid phase are:

$$
\hat{\sigma}^{f}=e^{-j \xi_{x} x}\left[\left(\chi+b_{1} \mu\right) D_{1} e^{-j \xi_{\alpha y} y}+\left(\chi+b_{1} \mu\right) D_{2} e^{j \xi_{\alpha y} y}+\left(\chi+b_{2} \mu\right) D_{3} e^{-j \xi_{\beta y} y}+\left(\chi+b_{2} \mu\right) D_{4} e^{j \xi_{\beta y} y}\right]
$$


where $\quad \xi_{\alpha y}=\sqrt{\xi_{\alpha}^{2}-\xi_{x}^{2}}, \quad \xi_{\beta y}=\sqrt{\xi_{\beta}^{2}-\xi_{x}^{2}}, \quad \xi_{t y}=\sqrt{\xi_{t}^{2}-\xi_{x}^{2}}, \quad j=\sqrt{-1}$, $b_{1}=\frac{\left(\hat{\rho}_{11} \mu-\hat{\rho}_{12} \chi\right)}{\left(\hat{\rho}_{22} \chi-\hat{\rho}_{12} \mu\right)}-\frac{\left(\phi \mu-\chi^{2}\right)}{\left[\omega^{2}\left(\hat{\rho}_{22} \chi-\hat{\rho}_{12} \mu\right)\right]} \xi_{\alpha}^{2}, \quad b_{2}=\frac{\left(\hat{\rho}_{11} \mu-\hat{\rho}_{12} \chi\right)}{\left(\hat{\rho}_{22} \chi-\hat{\rho}_{12} \mu\right)}-\frac{\left(\phi \mu-\chi^{2}\right)}{\left[\omega^{2}\left(\hat{\rho}_{22} \chi-\hat{\rho}_{12} \mu\right)\right]^{\prime}} \xi_{\beta}^{2}, \quad$ and $g=-\frac{\hat{\rho}_{12}}{\hat{\rho}_{22}}$.

These complex relations and six constants $D_{1}-D_{6}$ have to be determined by applying boundary conditions (BCs). When the elastic porous material is bonded directly to a panel, there exist six BCs. Also, the transverse displacement and in-plane displacement at the neutral axis can be followed as [1]:

$$
w_{t}(x, t)=W_{t}(x) e^{j \omega t} \text { and } w_{p}(x, t)=W_{p}(x) e^{j \omega t}
$$

$W_{t}(x)$ and $W_{p}(x)$ are transverse and in-plane displacements. Four BCs are obtained from the interface compatibility:

$$
V_{y}=j \omega W_{t}(x), \quad \hat{u}_{y}=W_{t}(x), \quad \hat{U}_{y}=W_{t}(x) \text { and } \hat{u}_{x}=W_{p}(x)(-/+) \frac{h_{p}}{2} \frac{d W_{t}(x)}{d x}
$$

where $h_{P}$ is the panel thickness and $V_{y}$ is the normal acoustic particle velocity. Two BCs are obtained from the equations of motion, followed as:

$$
\begin{gathered}
(+/-) \hat{p}_{a m b}(-/+) q_{P}-j \xi_{x} \frac{h_{P}}{2} \hat{\tau}_{x y}=\left(\hat{D} \xi_{x}^{4}-\omega^{2} I\right) W_{t}-\hat{B} \xi_{x}^{3} j W_{P} \\
(+/-) \hat{\tau}_{y x}=\left(\hat{A} \xi_{x}^{2}-\omega^{2} I\right) W_{P}-\hat{B} \xi_{x}^{3} j W_{t}
\end{gathered}
$$

$\hat{p}_{\text {amb }}$ is the acoustic pressures applied on the panel, $q_{P}$ is the normal force per unit panel area exerted on the panel by the elastic porous material $q_{P}=-\hat{\sigma}_{y}^{s}-\hat{\sigma}^{f}$. The Inertia term, $I$, and the extensional, coupling and bending stiffness, $\hat{A}, \hat{B}$ and $\hat{D}$ are [8]:

$$
\begin{gathered}
I=\sum_{l=1}^{L} \rho^{(l)}\left(y_{l}-y_{l-1}\right) \\
\hat{A}=\sum_{l=1}^{L} \hat{Q}^{(l)}\left(y_{l}-y_{l-1}\right) \\
\hat{B}=\frac{1}{2} \sum_{l=1}^{L} \hat{Q}^{(l)}\left(y_{l}^{2}-y_{l-1}^{2}\right)
\end{gathered}
$$




$$
\hat{D}=\frac{1}{3} \sum_{l=1}^{L} \hat{Q}^{(l)}\left(y_{l}^{3}-y_{l-1}^{3}\right)
$$

where $\rho^{(l)}$ is the mass density of the $l$ th layer of the shell per unit midsurface area and $y_{l}$ is the distance from the midsurface to the surface of the $l$ th layer having the farthest $y$ coordinate. The material constant $\hat{Q}^{(l)}$ is defined as $\hat{Q}^{(l)}=\frac{E_{1}^{(l)}}{1-v_{12} v_{21}}$ where $E_{1}^{(l)}$ is module of elasticity in the direction 1 , and $v_{12}^{(l)}$ and $v_{21}^{(l)}$ are Poisson's ratios in the directions 1 and 2 of the $l$ th ply, respectively. The fiber coordinates of ply is described, as 1 and 2, where direction 1 is parallel to the fibers and 2 is perpendicular to them. In BCs, the first signs are appropriate when the porous material is attached to the positive $y$ - facing surface of the panel, and the second signs when the porous material is attached to the negative $y$ - facing surface.

\section{Prediction of ratios of the energy}

The energy related to the waves in the fluid phase and solid phase are descript as follows [2].

The airborne wave:

$$
\begin{gathered}
E_{1 f}=\frac{1}{2}\left[\phi \cdot\left|\left(\chi+b_{1} \mu\right) \cdot b_{1} \frac{\xi_{\alpha y}^{2}}{\xi_{\alpha}^{2}} D_{1}^{2}\right|\right] \\
\left.E_{1 s}=\frac{1}{2}\left[(1-\phi) \cdot \mid 2 \delta \frac{\xi_{\alpha y}^{2}}{\xi_{\alpha}^{2}}+A+b_{1} \chi\right) \cdot \frac{\xi_{\alpha y}^{2}}{\xi_{\alpha}^{2}} D_{1}^{2} \mid\right]
\end{gathered}
$$

And the frame wave:

$$
\begin{gathered}
E_{3 f}=\frac{1}{2}\left[\phi \cdot\left(\chi+b_{2} \mu\right) \cdot b_{2} \frac{\xi_{\beta y}^{2}}{\xi_{\beta}^{2}} D_{3}^{2} \mid\right] \\
\left.E_{3 s}=\frac{1}{2}\left[(1-\phi) \cdot \mid 2 \delta \frac{\xi_{\beta y}^{2}}{\xi_{\beta}^{2}}+A+b_{2} \chi\right) \cdot \frac{\xi_{\beta y}^{2}}{\xi_{\beta}^{2}} D_{3}^{2} \mid\right] \\
\left.E_{5 s}=\frac{1}{2}|(1-\phi) \cdot| 2 \delta\left(\frac{\xi_{t y}^{2}}{\xi_{t}^{2}}\right)^{2} D_{5}^{2} \mid\right]
\end{gathered}
$$


where the subscripts $f$ and $s$ represents the fluid and solid phases, respectively.

\section{Formulation of the problem}

In the external space, the wave equation becomes [3]:

$$
c_{1} \nabla^{2}\left(p^{I}+p_{1}^{R}\right)+\frac{\partial^{2}\left(p^{I}+p_{1}^{R}\right)}{\partial t^{2}}=0
$$

where $p^{I}$ and $p_{1}^{R}$ are the acoustic pressures of the incident and reflected waves and $\nabla^{2}$ is the Laplacian operator in the cylindrical coordinate system, and $c_{1}$ is the speed of sound of outside medium. The wave equations in the fluid phase of the porous layer and internal space are the same as Eq. (33) with different variable names.

The shell motions are described by classic theory, fully considering the displacements in all three directions. Let the axial coordinate be $z$, the circumferential direction be $\theta$ and the normal direction to the middle surface of the shell be $r$. Equations of motion in the axial, circumferential and radial directions of a laminated composite thin cylindrical shell in cylindrical coordinate can be written as below [7]:

$$
\begin{gathered}
\frac{\partial N_{\alpha}}{\partial z}+\frac{1}{R_{i, e}} \frac{\partial N_{\alpha \beta}}{\partial \theta}+q_{\alpha_{i, e}}=-\widehat{I}_{i, e} \frac{\partial^{2} u_{i, e}^{0}}{\partial t^{2}} \\
\frac{\partial N_{\alpha \beta}}{\partial z}+\frac{1}{R_{i, e}} \frac{\partial N_{\beta}}{\partial \theta}+\frac{1}{R_{i, e}}\left[\frac{\partial M_{\alpha \beta}}{\partial z}+\frac{1}{R_{i, e}} \frac{\partial M_{\beta}}{\partial \beta}\right]+q_{\beta i, e}=-\widehat{I}_{i, e} \frac{\partial^{2} v_{i, e}^{0}}{\partial t^{2}} \\
\frac{\partial^{2} M_{\alpha}}{\partial z^{2}}-\frac{\partial N_{\beta}}{R_{i, e}}+2 \frac{1}{R_{i, e}} \frac{\partial^{2} M_{\alpha \beta}}{\partial \theta \partial z}+\frac{1}{R_{i, e}^{2}} \frac{\partial^{2} M_{\beta}}{\partial \theta^{2}}+q_{z_{i, e}}=-\widehat{I}_{i, e} \frac{\partial^{2} w_{i, e}^{0}}{\partial t^{2}}
\end{gathered}
$$

In which the subscripts $i$ and $e$ represent the inner and outer shells. $q_{\alpha}, q_{\beta}$, and $q_{z}$ are external pressure components. $u^{0}, v^{0}$, and $w^{0}$ are the displacements of the shell at the neutral surface in the axial, circumferential, and radial directions respectively. The inertia terms are followed as:

$$
\widehat{I}=\widehat{I}_{1}+\widehat{I}_{2}\left(\frac{1}{R}\right) \text { where }\left[\hat{I}_{1}, \widehat{I}_{2}\right]=\sum_{l=1}^{L} \int_{y_{l-1}}^{y_{l}} \rho^{(l)}[1, z] d z
$$

$R$ is cylindrical radius. Mid-surface strain and curvature can be expressed as: 


$$
\begin{gathered}
\varepsilon_{0 \alpha}=\frac{\partial u^{0}}{\partial z}, \varepsilon_{0 \beta}=\frac{1}{R}\left\{\frac{\partial v^{0}}{\partial \theta}+w^{0}\right\} \text { and } \gamma_{0 \alpha \beta}=\frac{\partial v^{0}}{\partial z}+\frac{1}{R} \frac{\partial u^{0}}{\partial \theta} \\
\kappa_{\alpha}=-\frac{\partial^{2} w^{0}}{\partial z^{2}}, \quad \kappa_{\beta}=\frac{1}{R^{2}}\left\{\frac{\partial v^{0}}{\partial \theta}-\frac{\partial^{2} w^{0}}{\partial \theta^{2}}\right\} \text { and } \kappa=\frac{1}{R}\left\{\frac{\partial v^{0}}{\partial z}-2 \frac{\partial^{2} w^{0}}{\partial z \partial \theta}\right\}
\end{gathered}
$$

The forces and moments are:

$$
\left[\begin{array}{c}
N_{\alpha} \\
N_{\beta} \\
N_{\alpha \beta} \\
M_{\alpha} \\
M_{\beta} \\
M_{\alpha \beta}
\end{array}\right]=\left[\begin{array}{llllll}
A_{11} & A_{12} & A_{16} & B_{11} & B_{12} & B_{16} \\
A_{21} & A_{22} & A_{26} & B_{21} & B_{22} & B_{26} \\
A_{61} & A_{62} & A_{66} & B_{61} & B_{62} & B_{66} \\
B_{11} & B_{12} & B_{16} & D_{11} & D_{12} & D_{16} \\
B_{21} & B_{22} & B_{26} & D_{21} & D_{22} & D_{26} \\
B_{61} & B_{62} & B_{66} & D_{61} & D_{62} & D_{66}
\end{array}\right]\left[\begin{array}{c}
\varepsilon_{0 \alpha} \\
\varepsilon_{0 \beta} \\
\gamma_{0 \alpha \beta} \\
\kappa_{\alpha} \\
\kappa_{\beta} \\
\kappa
\end{array}\right]
$$

where the extensional, coupling and bending stiffness, $A_{\tilde{p} \tilde{q}}, B_{\tilde{p} \tilde{q}}$ and $D_{\tilde{p} \tilde{q}}$ are:

$$
\begin{gathered}
A_{\tilde{p} \tilde{q}}=\sum_{l=1}^{L} Q_{\tilde{p} \tilde{q}}^{(l)}\left(y_{l}-y_{l-1}\right) \quad \tilde{p}, \tilde{q}=1,2,3 \\
B_{\tilde{p} \tilde{q}}=\frac{1}{2} \sum_{l=1}^{L} Q_{\tilde{p} \tilde{q}}^{(l)}\left(y_{l}^{2}-y_{l-1}^{2}\right) \quad \tilde{p}, \tilde{q}=1,2,3 \\
D_{\tilde{p} \tilde{q}}=\frac{1}{3} \sum_{l=1}^{L} Q_{\tilde{p} \tilde{q}}^{(l)}\left(y_{l}^{3}-y_{l-1}^{3}\right) \quad \tilde{p}, \tilde{q}=1,2,3
\end{gathered}
$$

$Q_{\tilde{p} \tilde{q}}^{(l)}$, material constant, is the function of physical properties of each ply. The displacement components of the inner and outer shell at an arbitrary distance $r$ from the midsurface along the axial, the circumferential and the radial directions are [8]:

$$
\begin{aligned}
& u_{e}^{0}=\sum_{n=0}^{\infty} u_{n e}^{0} \cos (n \theta) \exp \left[j\left(\omega t-\xi_{1 z} z\right)\right] \\
& v_{e}^{0}=\sum_{n=0}^{\infty} v_{n e}^{0} \sin (n \theta) \exp \left[j\left(\omega t-\xi_{1 z} z\right)\right] \\
& w_{e}^{0}=\sum_{n=0}^{\infty} w_{n e}^{0} \cos (n \theta) \exp \left[j\left(\omega t-\xi_{1 z} z\right)\right]
\end{aligned}
$$




$$
\begin{gathered}
u_{i}^{0}=\sum_{n=0}^{\infty} u_{n i}^{0} \cos (n \theta) \exp \left[j\left(\omega t-\xi_{3 z} z\right)\right] \\
v_{i}^{0}=\sum_{n=0}^{\infty} v_{n i}^{0} \sin (n \theta) \exp \left[j\left(\omega t-\xi_{3 z} z\right)\right] \\
w_{i}^{0}=\sum_{n=0}^{\infty} w_{n i}^{0} \cos (n \theta) \exp \left[j\left(\omega t-\xi_{3 z} z\right)\right]
\end{gathered}
$$

In Eqs. $(44-46) \xi_{1}$, wave number in the external medium, is defined as $\xi_{1}=\frac{\omega}{c_{1}}$, $\xi_{1 z}=\xi_{1} \sin \gamma, \xi_{1 r}=\xi_{1} \cos \gamma$ and in Eqs. (47 - 49) $\xi_{3}$, wave number in the internal cavity, is expressed as $\xi_{3}=\frac{\omega}{c_{3}}, \xi_{3 z}=\xi_{1 z}, \xi_{3 r}=\sqrt{\xi_{3}^{2}-\xi_{3 z}^{2}}$.

The boundary conditions at the two interfaces between the shells and fluid are [2]:

$$
\begin{gathered}
\frac{\partial}{\partial r}\left(p^{I}+p_{1}^{R}\right)=-s_{1} \frac{\partial^{2} w_{e}^{0}}{\partial t^{2}} @ r=R_{e} \\
\frac{\partial}{\partial r}\left(p_{2}^{T}+p_{2}^{R}\right)=-s_{2} \frac{\partial^{2} w_{e}^{0}}{\partial t^{2}} @ r=R_{e} \\
\frac{\partial}{\partial r}\left(p_{2}^{T}+p_{2}^{R}\right)=-s_{2} \frac{\partial^{2} w_{i}^{0}}{\partial t^{2}} @ r=R_{i} \\
\frac{\partial}{\partial r}\left(p_{3}^{T}\right)=-s_{3} \frac{\partial^{2} w_{i}^{0}}{\partial t^{2}} @ r=R_{i}
\end{gathered}
$$

$s_{1}$ and $s_{3}$ are densities of outside and inside acoustic media, respectively. $s_{2}$ is the equivalent density of the porous material and can be obtained from the Simplified method as suggested in [2]. The harmonic plane incident wave $p^{I}$ can be expressed in cylindrical coordinates as [2]:

$$
p^{I}(r, z, \theta, t)=p_{0} e^{j\left(\omega t-\xi_{1 z} z\right)} \sum_{n=0}^{\infty} \varepsilon_{n}(-j)^{n} J_{n}\left(\xi_{1 r} r\right) \cos (n \theta)
$$

where $p_{0}$ is the amplitude of the incident wave, $n=0,1,2,3, \ldots$ indicates the circumferential mode number, $\varepsilon_{n}=1$ for $n=0$ and 2 for $n=1,2,3, \ldots$, and $J_{n}$ is the Bessel function of the first kind of order $n$. 
Considering the circular cylindrical geometry, the pressures are expanded as:

$$
\begin{aligned}
& p_{1}^{R}(r, z, \theta, t)=e^{j\left(\omega t-\xi_{1} z\right)} \sum_{n=0}^{\infty} p_{n 1}^{R} H_{n}^{2}\left(\xi_{1 r} r\right) \cos (n \theta) \\
& p_{2}^{T}(r, z, \theta, t)=e^{j\left(\omega t-\xi_{2} z\right)} \sum_{n=0}^{\infty} p_{n 2}^{T} H_{n}^{1}\left(\xi_{2 r} r\right) \cos (n \theta) \\
& p_{2}^{R}(r, z, \theta, t)=e^{j\left(\omega t-\xi_{2 z} z\right)} \sum_{n=0}^{\infty} p_{n 2}^{R} H_{n}^{2}\left(\xi_{2 r} r\right) \cos (n \theta) \\
& p_{3}^{T}(r, z, \theta, t)=e^{j\left(\omega t-\xi_{3 z} z\right)} \sum_{n=0}^{\infty} p_{n 3}^{T} H_{n}^{1}\left(\xi_{3 r} r\right) \cos (n \theta)
\end{aligned}
$$

where $H_{n}^{1}$ and $H_{n}^{2}$ are the Hankel functions of the first and second kind of order $n$, $\xi_{2 z}=\xi_{3 z}=\xi_{1 z}$, and $\xi_{2 r}=\sqrt{\xi_{2}^{2}-\xi_{z}^{2}}$. The wave number, $\xi_{2}$, in the porous core can be obtained from the simplified method as suggested in [2].

Substitution of the expressions in the displacements of the shell (Eq. (44)-Eq.(49)) and the acoustic pressures (Eq. (54)-Eq.(58)) equations into six shell equations (Eq. (34)-Eq.(36)) and four boundary conditions (Eq. (50)-Eq.(53)) yields ten equations, which can be decoupled for each mode if the orthogonality between the trigonometric functions is utilized. These ten equations can be sorted into a form of a matrix equation as follow:

$$
[\mathbf{H}]\left\{u_{n i}^{0}, v_{n i}^{0}, w_{n i}^{0}, u_{n e}^{0}, v_{n e}^{0}, w_{n e}^{0}, p_{n 1}^{R}, p_{n 2}^{T}, p_{n 2}^{R}, p_{n 3}^{T}\right\}^{T}=\{\boldsymbol{A}\}
$$

where $[\mathbf{h}]$ is a $10 \times 10$ matrix with components given in Appendix and $\{\boldsymbol{A}\}$ is:

$$
\{\boldsymbol{\lambda}\}=\left\{0,0,0,0,0,-p_{0} \varepsilon_{n}(-j)^{n} J_{n}\left(\xi_{1 r} R_{e}\right) \xi_{1 r},-p_{0} \varepsilon_{n}(-j)^{n} \frac{d J_{n}\left(\xi_{1 r} R_{e}\right)}{d r} \xi_{1 r}, 0,0,0\right\}^{T}
$$

The ten unknown coefficients $p_{n 1}^{R}, p_{n 2}^{T}, p_{n 2}^{R}, p_{n 3}^{T}, u_{n e}^{0}, v_{n e}^{0}, w_{n e}^{0}, u_{n i}^{0}, v_{n i}^{0}$ and $w_{n i}^{0}$ are obtained in terms of $p_{0}$ with solving Eq. (59) for each mode $n$, which then can be substituted back into Eqs. (44) to (49) and (54) to (58) to find the displacements of the shell and the acoustic pressures in series forms.

\section{Calculation of transmission losses (TLs)}

The transmission coefficient, $\tau(\gamma)$, is the ratio of the amplitudes of the incident and transmitted waves. $\tau(\gamma)$ is obviously a function of the incidence angle $\gamma$ defined by [2]: 


$$
\tau(\gamma)=\sum_{n=0}^{\infty} \frac{\operatorname{Re}\left\{p_{n 3}^{T} H_{n}^{1}\left(\xi_{3 r} R_{i}\right)\left(j \omega w_{1 n}^{0}\right)^{*}\right\} s_{1} c_{1} \pi R_{i}}{\varepsilon_{n} R_{e} \cos (\gamma) p_{0}^{2}}
$$

where $\varepsilon_{n}=1$ for $n=0$ and $\varepsilon_{n}=2$ for $n=1,2,3, \ldots . \operatorname{Re}\{\}$ and the superscript $*$ represents the real part and the complex conjugate of the argument.

To consider the random incidences, $\tau(\gamma)$ can be averaged according to the Paris formula [9]:

$$
\overline{\boldsymbol{\tau}}=2 \int_{0}^{\gamma_{m}} \tau(\gamma) \sin \gamma \cos \gamma d \gamma
$$

where $\gamma_{m}$ is the maximum incident angle. Integration of Eq. (62) is conducted numerically by Simpson's rule. Finally, the average TLs is obtained as:

$$
T L_{a v g}=10 \log \frac{1}{\bar{\tau}}
$$

In the following, the averaged TLs of the structure is calculated in terms of the $1 / 3$ octave band for random incidences.

\section{Convergence algorithm}

Eqs. (44) to (49) and (54) to (58) are obtained in series form. Therefore, enough numbers of modes should be included in the analysis to make the solution converge. Therefore, an iterative procedure is constructed in each frequency, considering the maximum iteration number. Unless the convergence condition is met, it iterates again. When the TLs calculated at two successive calculations are within a pre-set error bound, the solution is considered to have converged. An algorithm for the calculation of TLs at each frequency is followed as:

\section{REPEAT}

$T L_{n}=10 \log \frac{1}{\bar{\tau}}$

Set $n=n+1$

UNTIL $\left|T L_{n+1}\right|-\left|T L_{n}\right|<10^{-6}$

\section{Numerical results}

Parametric numerical studies of transmission loss (TLs) are conducted for a double-walled composite laminated cylindrical shell lined with porous material specified as follows, considering 1/3 octave band frequency. Table 1 presents the geometrical and environmental properties of a sandwich cylindrical structure. Each layer of the laminated composite shells 
are made of graphite/epoxy, see Table 2 . The plies were arranged in a $\left[0^{\circ}, 45^{\circ}, 90^{\circ},-45^{\circ}, 0^{\circ}\right] \mathrm{s}$ pattern.

The results are verified by those investigated by the authors' previous work for an especial case in which the porous material properties go into fluid phase (In other world, the porosity is close to 1). The comparison of these results shown in Fig. 3, indicates a good agreement.

The calculated transmission loss for the laminated composite shell is compared with those of other authors for a special case of isotropic materials. In other word, in this model the mechanical properties of the lamina in all directions are chosen the same as an isotropic material such as Aluminum, and then the fiber angles are approached into zero. Fig. 4 compares the TL values of the special case of laminated composite walls obtained from present model and those of aluminum walls from Lee's study [2]. The results show an excellent agreement.

We are going to verify the model in behavior comparing the results of cylindrical shell in a case where the radius of the cylindrical shell becomes large or the curvature becomes negligible with the results of the flat plate done by Bolton [1] (See Fig. 5). It should be also noted that both structures sandwich a porous layer and have the same thickness. Although it is not expected to achieve the same results as the derivation of the shell equations is quite different comparing with derivation of plate equations, however, the comparison between the two curves indicate that they behave in a same trend in the broadband frequencies.

\begin{tabular}{lccccc}
\hline & Ambient & Outer Shell & Porous Core & Inner Shell & Cavity \\
\hline Material & Air & Composite & Porous Material & Composite & Air \\
Density $\left(\mathrm{kg} / \mathrm{m}^{3}\right)$ & 1.21 & - & - & - & 0.94 \\
Speed of Sound $(\mathrm{m} / \mathrm{s})$ & 343 & - & - & - & 389 \\
Radius $(\mathrm{mm})$ & - & 172.5 & - & 150 & - \\
Thickness $(\mathrm{mm})$ & - & 2 & 20 & 3 & - \\
Bulk Density of Solid & - & - & 30 & - & - \\
Phase* $\left(\mathrm{kg} / \mathrm{m}^{3}\right)$ & - & - & & & - \\
Bulk Young's Modulus & - & - & 800 & - & - \\
(kPa) & - & - & 0.4 & - & - \\
Bulk Poisson's Ratio (-) & - & - & 25000 & - & - \\
Flow Resistivity (MKs) & - & - & 7.8 & - & - \\
Tortuosity (-) & - & - & 0.9 & - & - \\
Porosity (-) & - & - & 0.265 & - & - \\
Loss Factor (-) & & &
\end{tabular}

* in vacuo.

Table 1. Geometrical and environmental properties [2] 


\begin{tabular}{lcc}
\hline & Graphite/epoxy & Glass/epoxy \\
\hline Axial Modulus (GPa) & 137.9 & 38.6 \\
Circumferential Modulus (GPa) & 8.96 & 8.2 \\
Shear Modulus (GPa) & 7.1 & 4.2 \\
Density (kg/m $\left.{ }^{3}\right)$ & 1600 & 1900 \\
Major Poisson's Ratio (-) & 0.3 & 0.26 \\
\hline
\end{tabular}

Table 2. Orthotropic properties [3]

Figure 6 indicates that whenever the radius of the shell descends, the TL of the shell is ascending in low frequency region. It is due to the fact that the flexural rigidity of the cylinder will be increased with reduction of shells radii. In addition, decreasing the radius of the shell leads to weight reduction and then in high frequency especially in Mass-controlled region the power transmission into the structure increases.

Figure 7 shows the effect of the composite material on TL. Materials chosen for the comparison are graphite/epoxy and glass/epoxy (Table 2). The figure shows that material must be chosen properly to enhance TL at Stiffness-control zone. The results represent a desirable level of TL at Stiffness-control zone (Lower frequencies) for graphite/epoxy. It is readily seen that, in higher frequency, as a result of density of materials, the TL curves are ascending. Therefore, the TL of glass/epoxy is of the highest condition in the Masscontrolled region.

It is well anticipated that increase of porous layer thickness leads to increase of TL. As illustrated in Fig. 8, a considerable increase due to thickening the porous layer is obtained. As it is well obvious from this figure, the weight increase of about $12 \%$ ( $h_{c}=60 \mathrm{~mm}$ ) and $25 \%\left(h_{c}=100 \mathrm{~mm}\right)$, the averaged TL values are properly increased about $35 \%$ and $60 \%$, respectively in broadband frequency. It is a very interesting result that can encourage engineers to use these structures in industries.

Figure 9 shows a comparison between the transmission loss for a ten-layered composite shell and an aluminum shell with the same radius and thickness. Since, the composite shell is stiffer than the aluminum one, its TL is upper than that of aluminum shell in the Stiffnesscontrolled region. However, as a result of lower density of composite shell, it does not appear to be effective as an aluminum shell in Mass-controlled region.

The effect of stacking sequence is shown in Fig. 10. Two patterns $\left[0^{\circ}, 90^{\circ}, 0^{\circ}, 90^{\circ}, 0^{\circ}\right]_{s}$ and $\left[90^{\circ}, 0^{\circ}, 90^{\circ}, 0^{\circ}, 90^{\circ}\right]_{s}$ are defined to designate stacking sequence of plies. The arrangements of layers are so effective on TLs curve, especially on Stiffness-controlled region. However, no clear discrepancy is depicted in Mass-controlled region. 


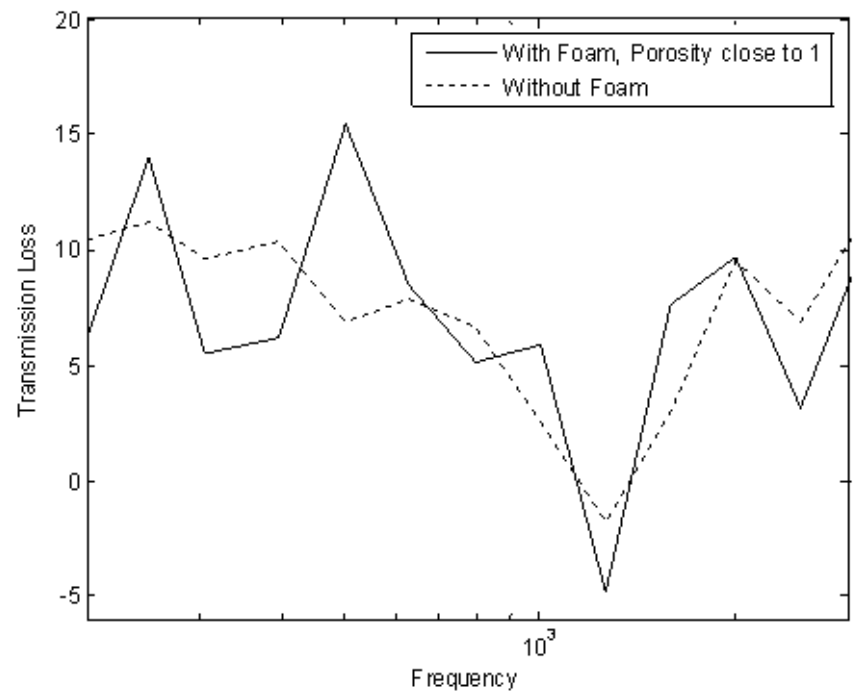

Figure 3. Comparison of cylindrical double-walled shell with and without foam where porosity close to 1

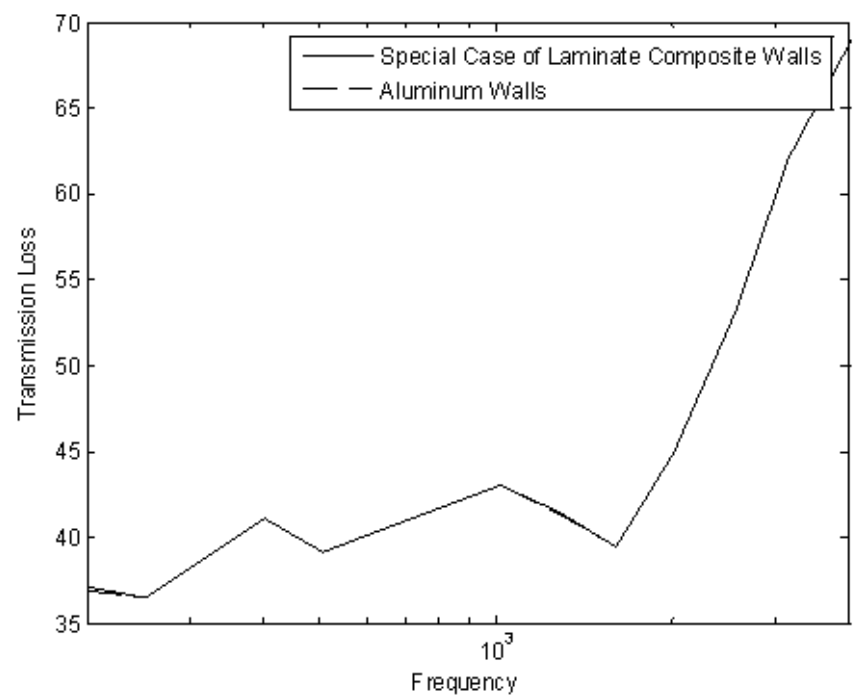

Figure 4. Comparison of cylindrical isotropic double-walled shell with special case of laminated composite double-walled shell $\left(R_{i}=150 \mathrm{~mm}, R_{e}=200 \mathrm{~mm}, h_{i}=3 \mathrm{~mm}, h_{e}=2 \mathrm{~mm}\right.$, and $\left.h_{c}=47.5 \mathrm{~mm}\right)$ 


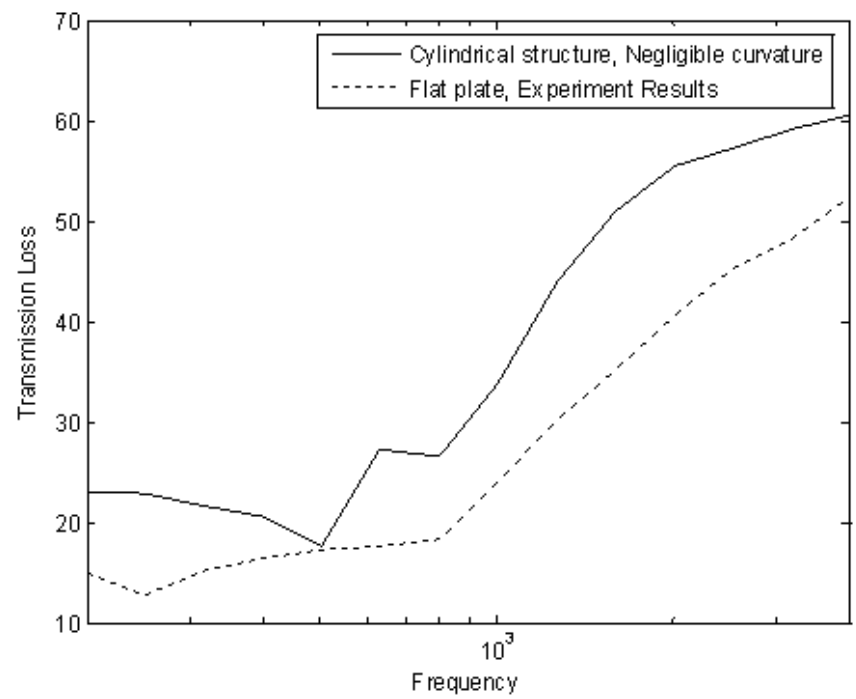

Figure 5. Comparison of an isotropic cylindrical double-walled shell with a negligible curvature and a double-panel structure

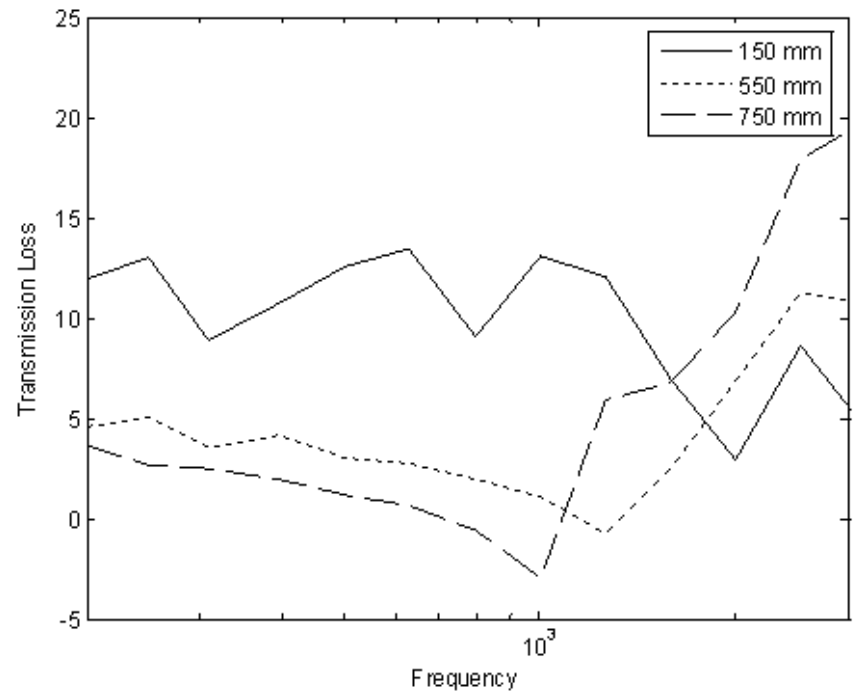

Figure 6. Comparison of a cylindrical double-walled shell lined with porous material with different inner shell's radius 


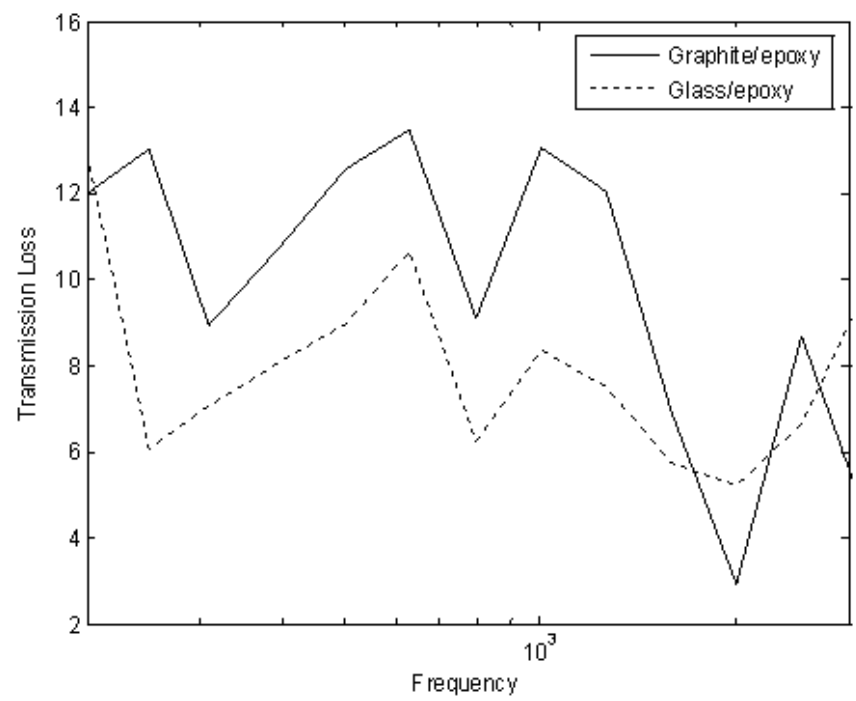

Figure 7. Comparison of a TL curves for ten-layer laminated composite shell with different material

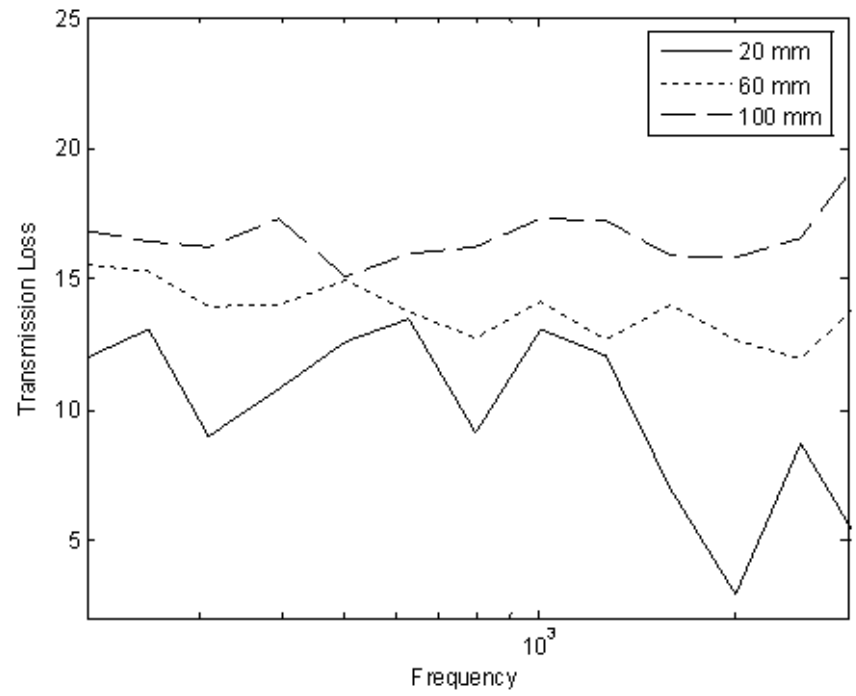

Figure 8. Comparison of a cylindrical double-walled shell lined with porous material with different core thickness 


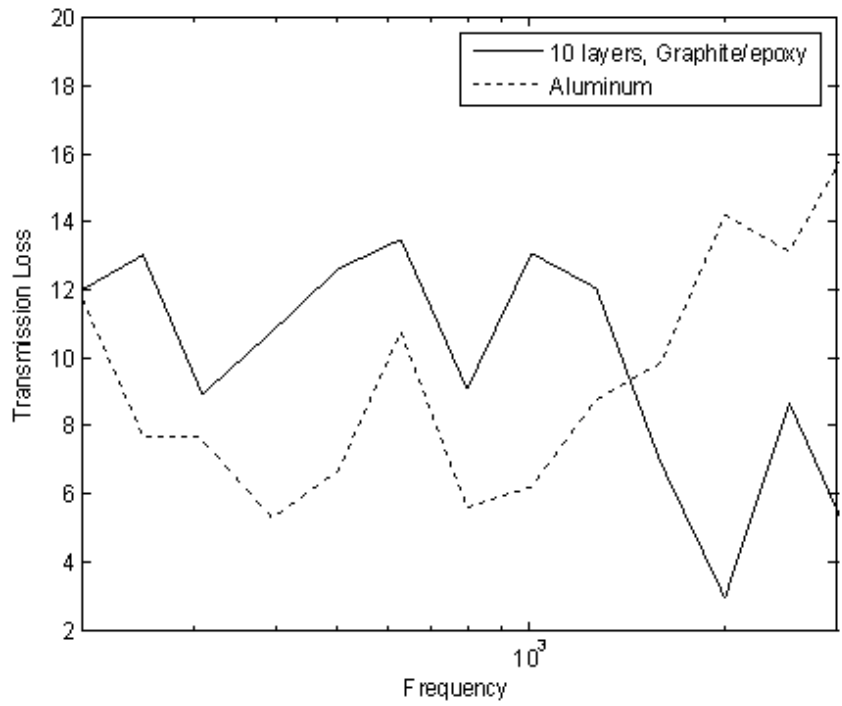

Figure 9. Comparison between Aluminum and ten-layer laminated composite shell

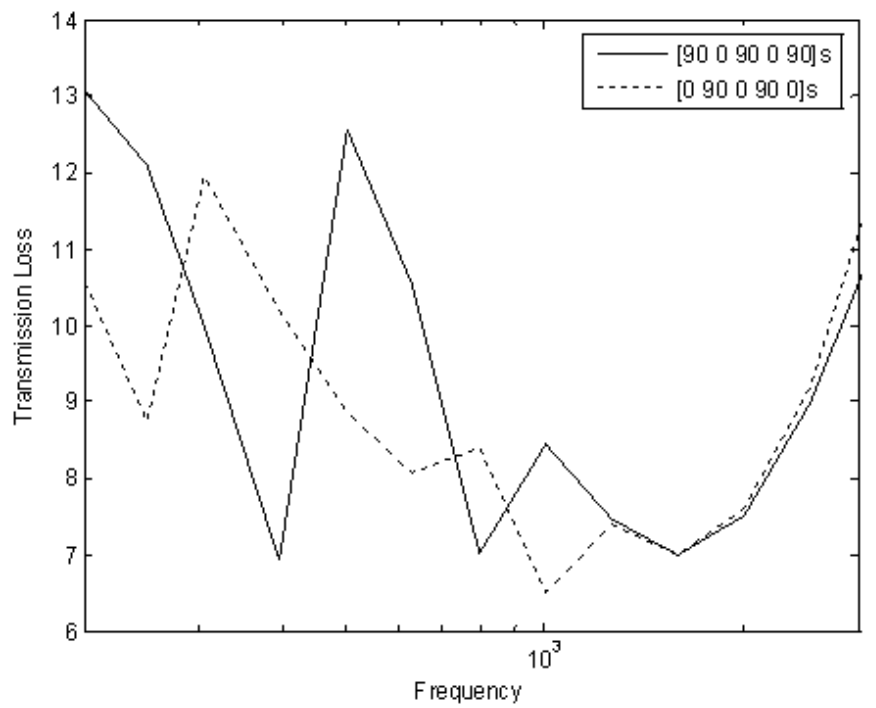

Figure 10. TL curves for the ten-layered composite shell with respect to stacking sequence

\section{Conclusions}

Transmission losses (TLs) of double-walled composite laminated shells sandwiching a layer of porous material were calculated. It is also considered the acoustic-structural coupling effect as well as the effect of the multi-waves in the porous layer. In order to make the 
problem solvable, one dominant wave was used to model the porous layer. In general the comparisons indicated the benefits of porous materials. Also, a considerable increase due to thickening the porous layer was obtained. For example, the weight increase of about $12 \%$ and $25 \%$ may respectively lead to an increase of $35 \%$ or $60 \%$ in amount of averaged TL values in broadband frequency. In addition, it was shown that increasing the axial modulus of plies made the TL be increased in low frequency range. Moreover, the comparison of double-walled shell with a gap and the one sandwiched with porous materials (where the porosity is close to 1) indicated a good agreement. Eventually the arrangement of layers in laminated composite can be so effective in Stiffness-controlled region. Therefore, optimizing the arrangement of layers can be useful in future study.

\section{Author details}

K. Daneshjou

Department of Mechanical Engineering, Iran University of Science and Technology, Tehran, Iran

H. Ramezani

Young Researchers Club, Islamic Azad University - Shahrerey branch, Tehran, Iran

R. Talebitooti

Department of Automotive Engineering, Iran University of Science and Technology, Tehran, Iran

\section{Nomenclature}

$\begin{array}{ll}A_{1}, A_{2} & \text { Physical and geometrical factors } \\ A_{\tilde{p} \tilde{q}}, B_{\tilde{p} \tilde{q}}, D_{\tilde{p} \tilde{q},}, \hat{A}, \hat{B}, \hat{D} & \text { Stiffness coefficients } \\ D_{1}-D_{6} & \text { Wave equations constants } \\ E & \text { Bulk Young's modulus } \\ E_{1}^{(l)} & \text { Module of elasticity in the direction } 1 \text { of the } l \text { th ply } \\ E_{1 f}, E_{1 s}, E_{3 s}, E_{3 f}, E_{5 s} & \text { The strain energy associated with the displacement in } \\ J_{n} & \text { the solid and fluid phases } \\ H_{n}^{1}, H_{n}^{2} & \text { Bessel function of the first kind of order } n \\ I, \hat{I}, \hat{I}_{1}, \hat{I}_{2} & \text { Hankel functions of the first and second kind of order } n \\ N_{\operatorname{Pr}} & \text { Inertia terms } \\ N_{\alpha}, N_{\beta}, N_{\alpha \beta}, M_{\alpha}, M_{\beta}, M_{\alpha \beta} & \text { Prandtl number } \\ \hat{Q}^{(l)}, Q_{\tilde{p} \tilde{q}}^{(l)} & \text { Forces and Moments } \\ R_{i}, R_{e} & \text { Material constant } \\ V_{y} & \text { Radii of inner and outer shell }\end{array}$




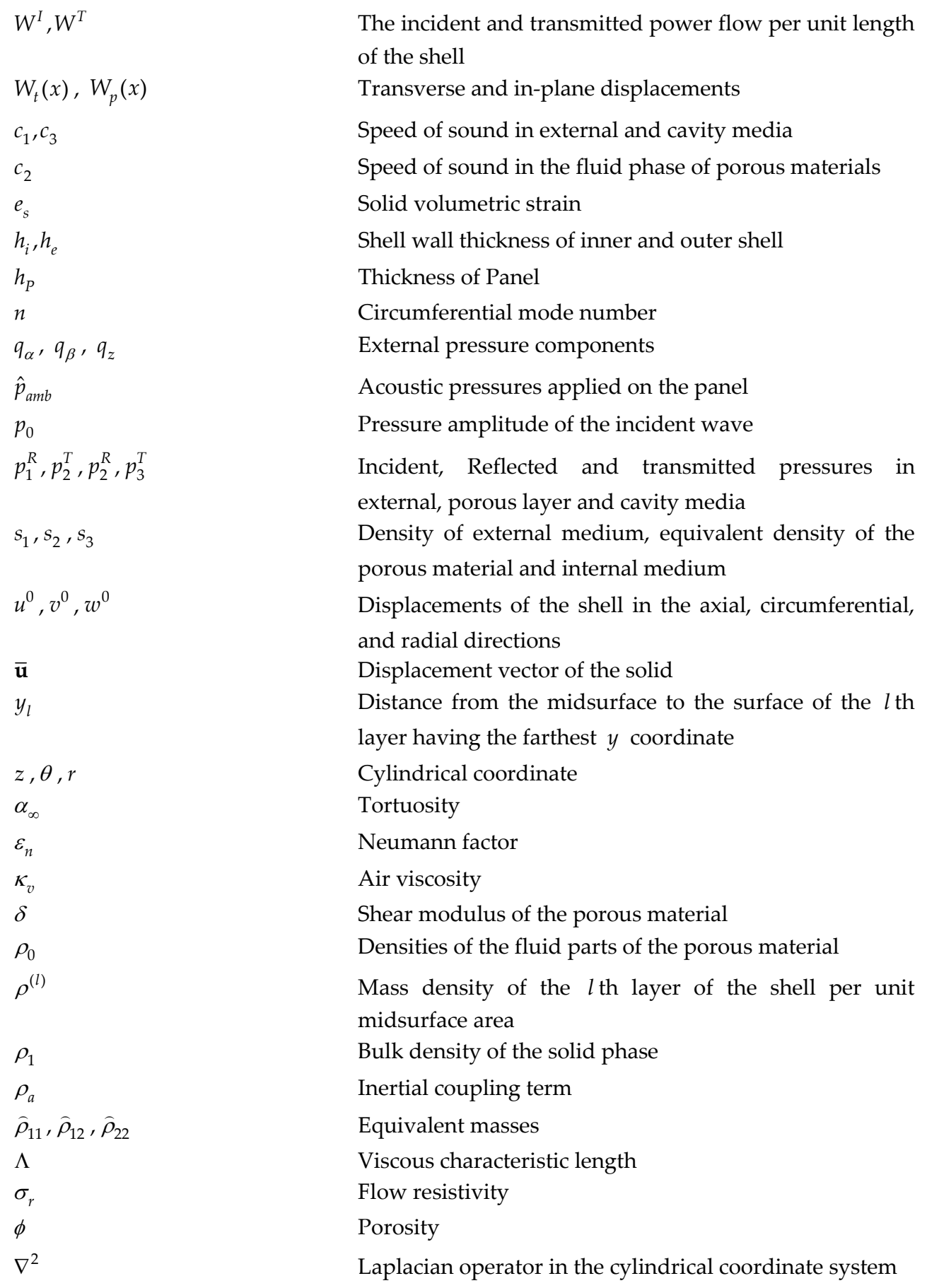

The incident and transmitted power flow per unit length of the shell

Transverse and in-plane displacements

Speed of sound in external and cavity media

Speed of sound in the fluid phase of porous materials

Solid volumetric strain

Shell wall thickness of inner and outer shell

Thickness of Panel

Circumferential mode number

External pressure components

Acoustic pressures applied on the panel

Pressure amplitude of the incident wave

Incident, Reflected and transmitted pressures in external, porous layer and cavity media

Density of external medium, equivalent density of the porous material and internal medium

Displacements of the shell in the axial, circumferential, and radial directions

Displacement vector of the solid

Distance from the midsurface to the surface of the $l$ th layer having the farthest $y$ coordinate

Cylindrical coordinate

Tortuosity

Neumann factor

Air viscosity

Shear modulus of the porous material

Densities of the fluid parts of the porous material

Mass density of the $l$ th layer of the shell per unit midsurface area

Bulk density of the solid phase

Inertial coupling term

Equivalent masses

Viscous characteristic length

Flow resistivity

Porosity

Laplacian operator in the cylindrical coordinate system 


$\gamma$
$\gamma_{m}$
$\chi, \varphi, \mu$
$\omega$
$\varpi$
$\xi_{1}, \xi_{3}$
$\xi_{\alpha}, \xi_{\beta}, \xi_{t}$
$\mathfrak{J}_{i}$
$\hat{u}_{x}, \hat{u}_{y}, \hat{U}_{x}, \hat{U}_{y}$
$\hat{\sigma}_{y}^{s}, \hat{\tau}_{x y}, \hat{\sigma}^{f}$
$v_{12}^{(l)}, v_{21}^{(l)}$
$\hat{v}$
$\hat{\eta}$
$\varsigma$
$\operatorname{Re}\{\}, *$

Angle of incidence

Maximum incident angle

Material properties

Angular frequency

Rotational strain in the solid phase

Wave number in external and cavity media

Complex wave numbers of the two compression and one shear waves

Potential of the incident wave, 2D dimension

Displacement components in the solid and fluid phases, 2D dimension

Stresses in the solid and fluid phases, 2D dimension

Poisson's ratios in the directions 1 and 2 of the $l$ th ply, 2D dimension

Bulk Poisson's ratio

Loss factor

Ratio of specific heats

Real part and the complex conjugate

\section{Appendix}

The non-zero components of the matrix $[\mathbf{h}]$ appearing in equation (59) are followed as:

$$
\begin{gathered}
\hbar_{1,1}=A_{11}\left(-j \xi_{z}\right)^{2}+2 A_{16} \frac{1}{R_{i}}\left(-j \xi_{z}\right)(-n)+A_{66} \frac{1}{R_{i}^{2}}\left(-n^{2}\right)-I_{i} \omega^{2} \\
\hbar_{1,2}=A_{12} \frac{1}{R_{i}}\left(-j \xi_{z}\right)(n)+A_{16}\left(-j \xi_{z}\right)^{2}+B_{12} \frac{1}{R_{i}^{2}}\left(-j \xi_{z}\right)(n)+B_{16} \frac{1}{R_{i}}\left(-j \xi_{z}\right)^{2}+A_{62} \frac{1}{R_{i}^{2}}\left(-n^{2}\right)+ \\
A_{66} \frac{1}{R_{i}}\left(-j \xi_{z}\right)(n)+B_{62} \frac{1}{R_{i}^{3}}\left(-n^{2}\right)+B_{66} \frac{1}{R_{i}^{2}}\left(-j \xi_{z}\right)(n) \\
\hbar_{1,3}=A_{12} \frac{1}{R_{i}}\left(-j \xi_{z}\right)-B_{11}\left(-j \xi_{z}\right)^{3}-B_{12} \frac{1}{R_{i}^{2}}\left(-j \xi_{z}\right)\left(-n^{2}\right)-3 B_{16} \frac{1}{R_{i}}\left(-j \xi_{z}\right)^{2}(-n)+ \\
A_{62} \frac{1}{R_{i}^{2}}(-n)-B_{62} \frac{1}{R_{i}^{3}}\left(n^{3}\right)-2 B_{66} \frac{1}{R_{i}^{2}}\left(-n^{2}\right)\left(-j \xi_{z}\right)
\end{gathered}
$$




$$
\begin{aligned}
& \hbar_{2,1}=A_{12} \frac{1}{R_{i}}\left(-j \xi_{z}\right)(n)+A_{16}\left(-j \xi_{z}\right)^{2}+B_{12} \frac{1}{R_{i}^{2}}\left(-j \xi_{z}\right)(n)+B_{16} \frac{1}{R_{i}}\left(-j \xi_{z}\right)^{2}+A_{62} \frac{1}{R_{i}^{2}}\left(-n^{2}\right)+ \\
& A_{66} \frac{1}{R_{i}}\left(-j \xi_{z}\right)(n)+B_{62} \frac{1}{R_{i}^{3}}\left(-n^{2}\right)+B_{66} \frac{1}{R_{i}^{2}}\left(-j \xi_{z}\right)(n) \\
& \hbar_{2,2}=A_{22} \frac{1}{R_{i}^{2}}\left(-n^{2}\right)+2 A_{26} \frac{1}{R_{i}}\left(-j \xi_{z}\right)(n)+2 B_{22} \frac{1}{R_{i}^{3}}\left(-n^{2}\right)+4 B_{26} \frac{1}{R_{i}^{2}}\left(-j \xi_{z}\right)(n)+A_{66}\left(-j \xi_{z}\right)^{2}+ \\
& 2 B_{66} \frac{1}{R_{i}}\left(-j \xi_{z}\right)^{2}+D_{22} \frac{1}{R_{i}^{4}}\left(-n^{2}\right)+2 D_{23} \frac{1}{R_{i}^{3}}\left(-j \xi_{z}\right)(n)+D_{33} \frac{1}{R_{i}^{2}}\left(-j \xi_{z}\right)^{2}-I_{i} \omega^{2} \\
& \hbar_{2,3}=A_{22} \frac{1}{R_{i}^{2}}(-n)-B_{12} \frac{1}{R_{i}}(-n)\left(-j \xi_{z}\right)^{2}-B_{22} \frac{1}{R_{i}^{3}}\left(n^{3}\right)-3 B_{23} \frac{1}{R_{i}^{2}}\left(-j \xi_{z}\right)\left(-n^{2}\right)+A_{62} \frac{1}{R_{i}}\left(-j \xi_{z}\right)+ \\
& -B_{61}\left(-j \xi_{z}\right)^{3}-2 B_{66} \frac{1}{R_{i}}(-n)\left(-j \xi_{z}\right)^{2}+B_{22} \frac{1}{R_{i}^{3}}(-n)-D_{21} \frac{1}{R_{i}^{2}}\left(-j \xi_{z}\right)^{2}(-n)-D_{22} \frac{1}{R_{i}^{4}}\left(n^{3}\right)- \\
& 3 D_{23} \frac{1}{R_{i}^{3}}\left(-j \xi_{z}\right)\left(-n^{2}\right)+B_{32} \frac{1}{R_{i}^{2}}\left(-j \xi_{z}\right)-D_{61} \frac{1}{R_{i}}\left(-j \xi_{z}\right)^{3}-2 D_{33} \frac{1}{R_{i}^{2}}\left(-j \xi_{z}\right)^{2}(-n) \\
& \hbar_{3,1}=A_{12} \frac{1}{R_{i}}\left(-j \xi_{z}\right)-B_{11}\left(-j \xi_{z}\right)^{3}-B_{12} \frac{1}{R_{i}^{2}}\left(-j \xi_{z}\right)\left(-n^{2}\right)-3 B_{16} \frac{1}{R_{i}}\left(-j \xi_{z}\right)^{2}(-n)+ \\
& A_{62} \frac{1}{R_{i}^{2}}(-n)+-B_{62} \frac{1}{R_{i}^{3}}\left(n^{3}\right)-2 B_{66} \frac{1}{R_{i}^{2}}\left(-n^{2}\right)\left(-j \xi_{z}\right) \\
& \hbar_{3,2}=A_{22} \frac{1}{R_{i}^{2}}(-n)-B_{12} \frac{1}{R_{i}}(-n)\left(-j \xi_{z}\right)^{2}-B_{22} \frac{1}{R_{i}^{3}}\left(n^{3}\right)-3 B_{23} \frac{1}{R_{i}^{2}}\left(-j \xi_{z}\right)\left(-n^{2}\right)+ \\
& A_{62} \frac{1}{R_{i}}\left(-j \xi_{z}\right)+-B_{61}\left(-j \xi_{z}\right)^{3}-2 B_{66} \frac{1}{R_{i}}(-n)\left(-j \xi_{z}\right)^{2}+B_{22} \frac{1}{R_{i}^{3}}(-n)- \\
& D_{21} \frac{1}{R_{i}^{2}}\left(-j \xi_{z}\right)^{2}(-n)-D_{22} \frac{1}{R_{i}^{4}}\left(n^{3}\right)-3 D_{23} \frac{1}{R_{i}^{3}}\left(-j \xi_{z}\right)\left(-n^{2}\right)+ \\
& B_{32} \frac{1}{R_{i}^{2}}\left(-j \xi_{z}\right)-D_{61} \frac{1}{R_{i}}\left(-j \xi_{z}\right)^{3}-2 D_{33} \frac{1}{R_{i}^{2}}\left(-j \xi_{z}\right)^{2}(-n) \\
& \hbar_{3,3}=A_{22} \frac{1}{R_{i}^{2}}-2 B_{21} \frac{1}{R_{i}}\left(-j \xi_{z}\right)^{2}-2 B_{22} \frac{1}{R_{i}^{3}}\left(-n^{2}\right)-4 B_{23} \frac{1}{R_{i}^{2}}\left(-j \xi_{z}\right)(-n)+-D_{11}\left(-j \xi_{z}\right)^{4}- \\
& 2 D_{12} \frac{1}{R_{i}^{2}}\left(-n^{2}\right)\left(-j \xi_{z}\right)^{2}+4 D_{16} \frac{1}{R_{i}}\left(-j \xi_{z}\right)^{3}(-n)+4 D_{62} \frac{1}{R_{i}^{3}}\left(-j \xi_{z}\right)\left(n^{3}\right)+ \\
& 4 D_{33} \frac{1}{R_{i}^{2}}\left(-j \xi_{z}\right)^{2}\left(-n^{2}\right)+D_{22} \frac{1}{R_{i}^{4}}\left(n^{4}\right)-I_{i} \omega^{2} \\
& \hbar_{3,8}=H_{n}^{1}\left(\xi_{2 r} R_{i}\right)_{,}, \hbar_{3,9}=H_{n}^{2}\left(\xi_{2 r} R_{i}\right), \hbar_{3,10}=H_{n}^{1}\left(\xi_{3 r} R_{i}\right)
\end{aligned}
$$




$$
\begin{aligned}
& \hbar_{4,4}=A_{11}\left(-j \xi_{z}\right)^{2}+2 A_{16} \frac{1}{R_{e}}\left(-j \xi_{z}\right)(-n)+A_{66} \frac{1}{R_{e}^{2}}\left(-n^{2}\right)-I_{e} \omega^{2} \\
& \hbar_{4,5}=A_{12} \frac{1}{R_{e}}\left(-j \xi_{z}\right)(n)+A_{16}\left(-j \xi_{z}\right)^{2}+B_{12} \frac{1}{R_{e}^{2}}\left(-j \xi_{z}\right)(n)+B_{16} \frac{1}{R_{e}}\left(-j \xi_{z}\right)^{2}+A_{62} \frac{1}{R_{e}^{2}}\left(-n^{2}\right)+ \\
& A_{66} \frac{1}{R_{e}}\left(-j \xi_{z}\right)(n)+B_{62} \frac{1}{R_{e}^{3}}\left(-n^{2}\right)+B_{66} \frac{1}{R_{e}^{2}}\left(-j \xi_{z}\right)(n) \\
& \hbar_{4,6}=A_{12} \frac{1}{R_{e}}\left(-j \xi_{z}\right)-B_{11}\left(-j \xi_{z}\right)^{3}-B_{12} \frac{1}{R_{e}^{2}}\left(-j \xi_{z}\right)\left(-n^{2}\right)-3 B_{16} \frac{1}{R_{e}}\left(-j \xi_{z}\right)^{2}(-n)+ \\
& A_{62} \frac{1}{R_{e}^{2}}(-n)+-B_{62} \frac{1}{R_{e}^{3}}\left(n^{3}\right)-2 B_{66} \frac{1}{R_{e}^{2}}\left(-n^{2}\right)\left(-j \xi_{z}\right) \\
& \hbar_{5,4}=A_{12} \frac{1}{R_{e}}\left(-j \xi_{z}\right)(n)+A_{16}\left(-j \xi_{z}\right)^{2}+B_{12} \frac{1}{R_{e}^{2}}\left(-j \xi_{z}\right)(n)+B_{16} \frac{1}{R_{e}}\left(-j \xi_{z}\right)^{2}+A_{62} \frac{1}{R_{e}^{2}}\left(-n^{2}\right)+ \\
& A_{66} \frac{1}{R_{e}}\left(-j \xi_{z}\right)(n)+B_{62} \frac{1}{R_{e}^{3}}\left(-n^{2}\right)+B_{66} \frac{1}{R_{e}^{2}}\left(-j \xi_{z}\right)(n) \\
& \hbar_{5,5}=A_{22} \frac{1}{R_{e}^{2}}\left(-n^{2}\right)+2 A_{26} \frac{1}{R_{e}}\left(-j \xi_{z}\right)(n)+2 B_{22} \frac{1}{R_{e}^{3}}\left(-n^{2}\right)+4 B_{26} \frac{1}{R_{e}^{2}}\left(-j \xi_{z}\right)(n)+A_{66}\left(-j \xi_{z}\right)^{2}+ \\
& 2 B_{66} \frac{1}{R_{e}}\left(-j \xi_{z}\right)^{2}+D_{22} \frac{1}{R_{e}^{4}}\left(-n^{2}\right)+2 D_{23} \frac{1}{R_{e}^{3}}\left(-j \xi_{z}\right)(n)+D_{33} \frac{1}{R_{e}^{2}}\left(-j \xi_{z}\right)^{2}-I_{e} \omega^{2} \\
& \hbar_{5,6}=A_{22} \frac{1}{R_{e}^{2}}(-n)-B_{12} \frac{1}{R_{e}}(-n)\left(-j \xi_{z}\right)^{2}-B_{22} \frac{1}{R_{e}^{3}}\left(n^{3}\right)-3 B_{23} \frac{1}{R_{e}^{2}}\left(-j \xi_{z}\right)\left(-n^{2}\right)+ \\
& A_{62} \frac{1}{R_{e}}\left(-j \xi_{z}\right)+-B_{61}\left(-j \xi_{z}\right)^{3}-2 B_{66} \frac{1}{R_{e}}(-n)\left(-j \xi_{z}\right)^{2}+B_{22} \frac{1}{R_{e}^{3}}(-n)- \\
& D_{21} \frac{1}{R_{e}^{2}}\left(-j \xi_{z}\right)^{2}(-n)-D_{22} \frac{1}{R_{e}^{4}}\left(n^{3}\right)-3 D_{23} \frac{1}{R_{e}^{3}}\left(-j \xi_{z}\right)\left(-n^{2}\right)+B_{32} \frac{1}{R_{e}^{2}}\left(-j \xi_{z}\right)- \\
& D_{61} \frac{1}{R_{e}}\left(-j \xi_{z}\right)^{3}-2 D_{33} \frac{1}{R_{e}^{2}}\left(-j \xi_{z}\right)^{2}(-n) \\
& \hbar_{6,4}=A_{12} \frac{1}{R_{e}}\left(-j \xi_{z}\right)-B_{11}\left(-j \xi_{z}\right)^{3}-B_{12} \frac{1}{R_{e}^{2}}\left(-j \xi_{z}\right)\left(-n^{2}\right)-3 B_{16} \frac{1}{R_{e}}\left(-j \xi_{z}\right)^{2}(-n)+ \\
& A_{62} \frac{1}{R_{e}^{2}}(-n)+-B_{62} \frac{1}{R_{e}^{3}}\left(n^{3}\right)-2 B_{66} \frac{1}{R_{e}^{2}}\left(-n^{2}\right)\left(-j \xi_{z}\right)
\end{aligned}
$$




$$
\begin{aligned}
& \hbar_{6,5}=A_{22} \frac{1}{R_{e}^{2}}(-n)-B_{12} \frac{1}{R_{e}}(-n)\left(-j \xi_{z}\right)^{2}-B_{22} \frac{1}{R_{e}^{3}}\left(n^{3}\right)-3 B_{23} \frac{1}{R_{e}^{2}}\left(-j \xi_{z}\right)\left(-n^{2}\right)+ \\
& A_{62} \frac{1}{R_{e}}\left(-j \xi_{z}\right)+-B_{61}\left(-j \xi_{z}\right)^{3}-2 B_{66} \frac{1}{R_{e}}(-n)\left(-j \xi_{z}\right)^{2}+B_{22} \frac{1}{R_{e}^{3}}(-n)- \\
& D_{21} \frac{1}{R_{e}^{2}}\left(-j \xi_{z}\right)^{2}(-n)-D_{22} \frac{1}{R_{e}^{4}}\left(n^{3}\right)-3 D_{23} \frac{1}{R_{e}^{3}}\left(-j \xi_{z}\right)\left(-n^{2}\right)+ \\
& B_{32} \frac{1}{R_{e}^{2}}\left(-j \xi_{z}\right)-D_{61} \frac{1}{R_{e}}\left(-j \xi_{z}\right)^{3}-2 D_{33} \frac{1}{R_{e}^{2}}\left(-j \xi_{z}\right)^{2}(-n) \\
& \hbar_{6,6}=A_{22} \frac{1}{R_{e}^{2}}-2 B_{21} \frac{1}{R_{e}}\left(-j \xi_{z}\right)^{2}-2 B_{22} \frac{1}{R_{e}^{3}}\left(-n^{2}\right)-4 B_{23} \frac{1}{R_{e}^{2}}\left(-j \xi_{z}\right)(-n)+ \\
& -D_{11}\left(-j \xi_{z}\right)^{4}-2 D_{12} \frac{1}{R_{e}^{2}}\left(-n^{2}\right)\left(-j \xi_{z}\right)^{2}+4 D_{16} \frac{1}{R_{e}}\left(-j \xi_{z}\right)^{3}(-n)+ \\
& 4 D_{62} \frac{1}{R_{e}^{3}}\left(-j \xi_{z}\right)\left(n^{3}\right)+4 D_{33} \frac{1}{R_{e}^{2}}\left(-j \xi_{z}\right)^{2}\left(-n^{2}\right)+D_{22} \frac{1}{R_{e}^{4}}\left(n^{4}\right)-I_{e} \omega^{2} \\
& \hbar_{6,7}=H_{n}^{2}\left(\xi_{1 r} R_{e}\right), \quad \hbar_{6,8}=H_{n}^{1}\left(\xi_{2 r} R_{e}\right), \quad \hbar_{6,9}=-H_{n}^{2}\left(\xi_{2 r} R_{e}\right), \quad \hbar_{7,6}=-s_{1} \omega^{2} \\
& \hbar_{7,7}=H_{n}^{2^{\prime}}\left(\xi_{1 r} R_{e}\right) \xi_{1 r}, \quad \hbar_{8,6}=-S_{2} \omega^{2}, \quad \hbar_{8,8}=H_{n}^{1^{\prime}}\left(\xi_{2 r} R_{e}\right) \xi_{2 r}, \quad \hbar_{8,9}=p_{n 2}^{R} H_{n}^{2^{\prime}}\left(\xi_{2 r} R_{e}\right) \xi_{2 r} \\
& \hbar_{9,3}=-s_{2} \omega^{2}, \quad \hbar_{9,8}=H_{n}^{1^{\prime}}\left(\xi_{2 r} R_{i}\right) \xi_{2 r}, \quad \hbar_{9,9}=H_{n}^{2^{\prime}}\left(\xi_{2 r} R_{i}\right) \xi_{2 r}, \quad \hbar_{10,3}=-s_{3} \omega^{2}, \\
& \hbar_{10,10}=H_{n}^{1^{\prime}}\left(\xi_{3 r} R_{i}\right) \xi_{3 r}
\end{aligned}
$$

Here:

$$
()^{\prime}=\frac{d}{d r}, \quad \xi_{z}=\xi_{1 z}=\xi_{2 z}=\xi_{3 z}, \quad \xi_{2 r}=\sqrt{\xi_{2}^{2}-\xi_{z}^{2}} .
$$

\section{References}

[1] Bolton J S, Shiau N M, Kang Y J (1996) Sound Transmission through Multi-Panel Structures Lined with Elastic Porous Materials. Journal of Sound and Vibration. 191: 317-347.

[2] Lee J H, Kim J (2001) Simplified method to solve sound transmission through structures lined with elastic porous material. J. of the Acoust. Soc of Am. 110(5): 2282-2294.

[3] Daneshjou K, Nouri A, Talebitooti R (2006) Sound transmission through laminated composite cylindrical shells using analytical model. Arch. Appl. Mech. 77: 363-379.

[4] Daneshjou K, Talebitooti R, Nouri A (2007) Analytical Model of Sound Transmission through Orthotropic Double Walled Cylindrical Shells. CSME Transaction. 32(1). 
[5] Daneshjou K, Talebitooti R, Nouri A (2009) Investigation model of sound transmission through orthotropic cylindrical shells with subsonic external flow. Aerospace Science and Technology. 13: 18-26.

[6] Daneshjou K, Ramezani H, Talebitooti R (2011) Wave Transmission through Laminated Composite Double-Walled Cylindrical Shell Lined with Porous Materials. Applied Mathematics and Mechanics - English Edition, 32(6): 1-16.

[7] Qatu M S (2004)Vibration of Laminated Shells and Plates. Amsterdam: Elsevier Academic.

[8] Leissa A (1973) Vibration of Shells. Scientific and technical Information center, NASA, Washington D.C.

[9] Pierce A D (1981) Acoustics. New York: McGraw-Hill. 